OBETS. Revista de Ciencias Sociales

Vol. 16, n 2, 2021, pp. 297-314

ISSN: 1989-1385

https://doi.org/10.14198/OBETS2021.16.2.05

\title{
REPRESENTACIÓN ESCOLAR DE LOS ESPACIOS RURALES: UNA INVESTIGACIÓN DIDÁCTICA MEDIANTE MÉTODOS MIXTOS ${ }^{1}$
}

\author{
SCHOOL REPRESENTATION OF RURAL SPACES: A DIDACTIC RESEARCH \\ THROUGH MIXED METHODS
}

\author{
Diego García Monteagudo \\ Universitat de València, España \\ Diego.Garcia-Monteagudo@uv.es \\ https://orcid.org/0000-0003-0505-0608
}

\begin{abstract}
Cómo citar / Citation: García, D. (2021) "Representación escolar de los espacios rurales: una investigación didáctica mediante métodos mixtos". OBETS. Revista de Ciencias Sociales, 16(2): 297-314. https://doi.org/10.14198/OBETS2021.16.2.05
\end{abstract}

\section{(C) 2021 Diego García Monteagudo}

Este es un artículo de acceso abierto distribuido bajo los términos de la licencia de uso y distribución Creative Commons Reconocimiento 4.0 Internacional (CC BY 4.0) https://creativecommons.org/licenses/by/4.0/deed.es

Recibido: 26/02/20. Aceptado: 31/12/20

\section{Resumen}

Los espacios rurales son objeto de representación histórica desde un relato homogéneo que no ha sido cuestionado en la geografía escolar y afecta a las explicaciones que realiza el alumnado de Educación Secundaria y Bachillerato. La teoría de las representaciones sociales y su relación con el espacio subjetivo permiten comprender las concepciones escolares del alumnado de diez centros escolares de la provincia de Valencia (España) acerca de los espacios rurales. Mediante un enfoque dual (estructural y procesual) que se acompaña de técnicas cualitativas y cuantitativas aplicadas al campo de la didáctica de las ciencias sociales se pretende diagnosticar los elementos que forman la cosmovisión que tiene el alumnado, como resultado de una representación social que idealiza estos espacios.

Palabras clave: Espacios rurales; Relato homogéneo; Representación idealizada; Alumnado; Enfoque dual; Cosmovisión.

\begin{abstract}
Rural spaces are object of historical representation from a homogeneous account that has not been questioned in the school geography and affects the explanations made by the students of Secondary Education and Baccalaureate. The theory of social representations and their relationship with the subjective space allow us to understand the students school conceptions of ten schools in the province of Valencia (Spain) about rural spaces. Through a dual approach (structural and procedural) that is accompanied by qualitative and quantitative techniques applied to the field of social science teaching, it is intended to diagnose the elements that make up the worldview that students have, as a result of a social representation that idealizes these spaces.
\end{abstract}

Keywords: Rural spaces; Homogeneous story; Idealized representation; Students; Dual focus; Worldview.

1 Este trabajo forma parte del proyecto de investigación "Las representaciones sociales de los contenidos escolares en el desarrollo de las competencias docentes" (PGC2018-094491-B-C32), financiado por el Ministerio de Ciencia, Innovación y Universidades y cofinanciado con fondos FEDER de la UE. 


\section{Extended abstract}

The application of the theory of social representations to the didactic research of rural spaces is not a common perspective in the teaching of social sciences. In Spain, specialists in rural geography have not been interested in teaching rural spaces at pre-university levels (Ruiz, Tula \& Molinero, 2017) although it is one of the oldest branches of geographical science. The teaching of geography does not have too many specialists at international level and studies have hardly been devoted to the teaching of rural spaces (Matthews et al. 2000; Araya, Souto \& Herrera, 2015; Armas, Rodríguez \& Macía, 2018). The low relationship between the topics that are researched in the academic rural geography and its didactic transposition to the related to school (Rodríguez, 2002), through descriptive contents that do not favor the understanding of social and environmental problems in rural areas, makes it difficult to question a story that attributes a symbolic value to the rural and identifies it with the countryside (Entrena, 1998). This approach has been formed in the social sciences (Garayo, 1996) and has explained the rural ways of life as opposed to the urban or exclusive of the people who inhabit the cities (Williams, 2001). Therefore, the sample of students (393 students in 3rd ESO and 181 in 2nd year of Baccalaureate) comes from ten schools in rural, peri-urban and urban areas of the province of Valencia (Spain), as this allows us to know the influence of the context in the scholar explanation of these spaces.

The theoretical and methodological use of social representations (Moscovici, 1978) focused on the didactic research of rural spaces is based on the turn of behavior (Capel, 1973) from the humanistic side of the geography of perception and behavior (Souto, 2018). This is intended to demonstrate that there is an area of human behavior with respect to common sense ideas that explain rural spaces based on reasoning based on the comparison with urban spaces, including through the use of stereotypes (lower degree of rural civilization, support of the rural economy for the support of public policies and the emotional link with natural idealization) that have been compiled in some scientific publications (Romero \& Farinós, 2004; Molino, 2016). To contribute to a renewed and updated education, the subjective aspect of rural spaces needs to be introduced to facilitate its understanding as multiscale and multifunctional scenes (Hudault, 2011), in which the experiences and problems of its inhabitants are combined, with perceptions that condition social behavior and the influence of territorial management. This is how the main research questions have been constructed, taking as reference the interpretation that Souto (2018) makes of the spatial categories (lived space, perceived space and conceived space) of Soja (2008). The lived space explains the identity of the rural spaces by the emotional bonding that the students have that have direct contact with these spaces, while the perceived space resembles the "themata" concept that filters the conceptions that emanate from other sources of information that they influence the representation of the rural. The conceived space is related to the teaching of the geographical space in an objective sense, following the influence that the French regional geography has had in
Spain (Mata, 1987; Ortega, 2000) and the inclusion of the local scale in the teaching of the medium (Luis $\&$ Urteaga, 1982; Romero \& Luis, 2008).

With the didactic study of social representations, the relationships between students and the social context in which they develop their life and learning are reflected, in the context of the province of Valencia (Spain). Although learning is an individual process, it is influenced by the social and cultural context, so that social constructivism links the theory of social representations with the geography of perception and behavior. Knowledge is generated in society and allows us to know human behavior from social representations, since the stimulus generated by a social representation justifies a certain response according to a social representation that describes that object of study in an intelligible way for each person. The consequence is that the activity is carried out in a specific space (Sammut et al. 2015), in this case, in the behavior that the students of 3rd ESO and 2nd Baccalaureate have on the learning of rural spaces in schools and when traveling to rural spaces. This behavior can be mediated by the information that since historic times has reduced rural spaces to natural spaces. The naturalist current and the traditional literature began this story that affected rural drama (Pedraza, 2011) and some stereotypes have been represented in rural cinema (González, 1988; Crespo \& Quirosa, 2014). Subsequently, they have been taken in commercial advertising (Arranz, 2014) and reproduce a natural, calm and isolated image of rural spaces that influence the perception of young people (Pérez and Sánchez, 2007). In addition, the contents of the textbooks present rural spaces as producers of raw materials (Sánchez, 1998; Armas, Rodríguez \& Macía, 2018; Morón $\&$ Morón, 2019), and appear separated from urban spaces, which makes it difficult to understanding of processes that affect both spaces equally (Prados, 2009; Esparcia, 2012). The alternative is the didactic innovation projects that offer a closer look at the experiences and problems of rural spaces (D`Angelo \& Lossio, 2011; San Pedro \& López, 2017; Claudino et al. 2019).

The importance of representation in the context has been considered in the selection of the student sample. The 581 students belong to ten schools in the province of Valencia according to the classification established in the Valencian Territorial Strategy 2030, in which the territory is divided into the rural system, the intermediate strip of the territory and the level 100. Each student has answered a mixed questionnaire with varied questions that follow the spatial trilogy (lived space, perceived space and conceived space) with an initial section as a test of association of ideas (Agüero \& Chama, 2009; Navia \& Estrada, 2012). In addition, twenty-eight textbooks of these courses have been selected between 1959 and 2016 to learn about the school account about rural spaces and interviews and discussion groups have been conducted to students to understand their explanation about these spaces. The variety of data collection instruments is explained by the use of the structural and procedural approaches recommended by Banchs (2000) in the study of social representations through 
a mixed sequential exploratory method (Creswell, 2014). Subsequently, they have been triangulated (Flick, 2004; Okuda-Benavides \& Gómez-Restrepo, 2005) to respond to the main objective of the study: to know the elements that influence the representation that students in the province of Valencia have on rural spaces, since the context can influence the worldview they have about these spaces based on their consideration as lived, perceived or conceived spaces, with a different degree of idealization.

In the case of the structural approach, the word association questionnaire model includes three questions about the rural environment, following the Abric (2001) representative content research model interpreted by Brazilian authors (Domingos, 2000; Saraiva, 2007) and applied to similar studies (Lopes, 2010). The quantitative approach is included in the counting of the activities of the textbooks and their cognitive level (Sáiz \& Colomer, 2014), the photographs in which the human figure appears and the pictorial representations in which the presence of persons. In the photographs and drawings of the students, the chi-square test has been applied to assess whether the human presence is significant in the records of both courses.

For the procedural approach, students' drawings have been classified according to landscape functions (Hudault, 2011) and their content has been analyzed (Ortega, 2000). In the textbooks the most relevant contents have been analyzed and finally semi-structured interviews or discussion groups have been conducted with 58 students to learn their explanation about rural spaces.

The results show that the social representation that idealizes rural spaces and manifests itself in the different registers analyzed influences an idealized conception of students. The diagnosis will continue with the debates on the teaching of rural spaces that occur in the Ibero-American Geoforo on Education, Geography and Society.

\section{INTRODUCCIÓN}

La consideración de los espacios rurales como objeto de representación social tiene una larga tradición que trasciende el marco de su enseñanza como contenido predilecto de la geografía. El carácter multidisciplinar del concepto de paisaje se ha utilizado desde las ciencias sociales como símbolo de identidad (Gómez, 2008) y los discursos literarios y artísticos que se han proyectado sobre los paisajes rurales han cobrado una importancia capital en la cosmovisión de la vida en estos espacios (Tesser, 2000; Marco, 2012). La representación de escenas naturales a partir del Renacimiento y el énfasis otorgado a los elementos estéticos proporcionaron mayor relevancia a la contemplación de la naturaleza, lo que condiciona el filtro que supone la percepción humana sobre el paisaje (Maderuelo, 1996).

La cosmovisión que se forma sobre los territorios desde los organismos de gobierno se asemeja al paisaje político y contrasta con el paisaje vernacular. Este segundo concepto corresponde a la imagen que se forja desde las vivencias de las personas que forman parte de una unidad territorial (Gómez, 2013) y en cuyo análisis se combinan los espacios subjetivos con los espacios absolutos desde una vertiente posmodernista (Pillet, 2004). De ambas tipologías de paisaje resulta un espacio complejo propio de la geografía de la percepción y del comportamiento. De hecho el espacio complejo que constituye el medio rural en esta investigación es similar al ámbito de las representaciones sociales,

pues las ideas sobre estos espacios se construyen en un contexto social determinado en el que se plasma el comportamiento de las personas (Sammut et al. 2015). La interpretación didáctica de la trialéctica espacial de Soja (2008) realizada por Souto (2018) es pertinente para abordar las concepciones escolares de los espacios rurales desde la teoría de las representaciones sociales, ya que este paradigma es el que más se relaciona con dichos espacios (Cloke, 2006). Este diagnóstico escolar sobre lo rural no es demasiado frecuente en la didáctica de las ciencias sociales (Araya, Souto y Herrera, 2015; García-Monteagudo, 2018), por lo que comenzaremos exponiendo algunas líneas relevantes sobre la construcción de los contenidos rurales en el ámbito didáctico. Posteriormente se defenderá la necesidad de emplear métodos mixtos que permitan acceder a la representación escolar de estos espacios entre el alumnado de Educación Secundaria y Bachillerato de la provincia de Valencia. Por todo ello, lo que se pretende es diagnosticar los elementos que influyen en la representación escolar de los espacios rurales y comprobar si se corresponden con una representación social más amplia que puede condicionar las propuestas de innovación desde el ámbito de la geografía escolar.

\subsection{La didáctica de los espacios rurales en la encrucijada: las dificultades de impugnar un relato idealizado desde la geografía escolar}

La investigación didáctica de los espacios rurales no ha sido objeto de interés destacado por los geógrafos 
españoles, que han dedicado más esfuerzo al campo de la geografía rural (Ruiz, Tula y Molinero, 2017). En el plano académico, la geografía ha estudiado el paisaje agrario desde un marco ecológico que ha seguido los postulados de la geografía regional (Estébanez, 1986; Ortega, 2000). La influencia francesa sobre la investigación española se tradujo en estudios de geografía agraria en los que se analizaban los modos de vida y las formas de asentamiento de la población rural (George, 1973; Chapuis, 1995). La geografía rural surgió tras la Segunda Guerra Mundial, pero el peso que tuvo la herencia francesa en nuestro país derivó en el mantenimiento de estudios sobre la reconstrucción histórica de los paisajes agrarios, los sistemas de explotación y propiedad de la tierra, y la transformación de cultivos (Mata, 1987). Así, otras temáticas como la diversidad de funciones de los espacios rurales, el desarrollo agroindustrial y la calidad del medio ambiente han llegado más tardíamente a la investigación española (García, 1992; Segrelles, 2002; Paniagua, 2004).

Los contenidos enseñados en la geografía escolar tienen una estrecha relación con la investigación académica, pero la crítica a la perspectiva de la transposición didáctica revela que los primeros no son los únicos en la formación de la cultura escolar (Rodríguez, 2002). De hecho, los currículos escolares son el foco de encuentro entre intereses, enfoques e ideologías donde se sintetizan las concepciones de los docentes (Puente, 2001), cuyas estrategias didácticas reproducen una enseñanza más centrada en las identidades, costumbres y tradiciones de los espacios rurales, que en los problemas socialmente relevantes (Muñoz, 2012). Así se entiende la necesidad de recurrir al paradigma socioconstructivista que enfatiza el papel del aprendizaje en el desarrollo del pensamiento conceptual de los adolescentes y expone que el conocimiento escolar surge de la confrontación entre los conceptos científicos y los conceptos cotidianos (Cavalcanti, 2010). Esto significa que para el caso de la geografía rural escolar deben articularse contenidos que versen sobre la multifuncionalidad de los espacios rurales (Hudault, 2011) desde una vertiente sistémica, ya que así se garantizará un desarrollo rural sostenible que englobe la producción de alimentos y materias primas, así como la protección de la biodiversidad y de los valores paisajísticos, que asegurarán la prestación de servicios de producción, ambientales y culturales (Vilches, Gil, Toscano y Macías, 2014).
La tradición pedagógica de la enseñanza del medio es otro obstáculo que ha favorecido el enfoque de la geografía regional en el ámbito escolar. La influencia de Dewey, Cousinet y Freinet para construir una corriente de pensamiento y una práctica didáctica que vinculase el estudio del medio a la enseñanza de las ciencias sociales (Vilarrasa, 2005) se vio truncada con la incorporación del principio de la geografía local que asimila el espacio rural al medio en un sentido objetivo (Luis y Urteaga, 1982; Romero y Luis, 2008). En la práctica, los contenidos de los espacios rurales aparecen separados de los correspondientes a los espacios urbanos en los libros de texto, lo que supone limitar la comprensión de algunos procesos (la urbanización hacia zonas periféricas, el envejecimiento demográfico y la penetración de inmigración extranjera) que suceden tanto en unos espacios como en otros (Prados, 2009; Esparcia, 2012). Los contenidos de los libros de texto iberoamericanos tienen un carácter conservador e inducen a la memorización (Tonini, Claudino y Souto, 2015), que para el caso de los espacios rurales adquieren una visión economicista que los reduce al estudio de las actividades del sector primario (Sánchez, 1998; Armas, Rodríguez y Macía, 2018).

Frente a la escasa investigación didáctica de los espacios rurales se ha difundido un relato que encarna una representación positiva de lo rural, aun cuando persiste la idea de sumisión del campo a la ciudad (Entrena 1998; Paniagua y Hoggart, 2002). En esa representación virtual se entremezclan valores medioambientales y otros más vinculados a estilos de vida tranquilos y sosegados que solo existen en el imaginario social (Williams, 2001; Romero y Farinós, 2004). Desde época histórica la corriente naturalista y el costumbrismo regionalista de la literatura calaron en el drama rural (Pedraza, 2011), que se inició en España con el teatro de finales del siglo XIX (Espín, Vega y Lagos, 2016). Otras narrativas literarias han sustentado las apologías por los espacios rurales, otorgándole una difusión creciente al mito de la civilización contra la barbarie (Fusi, 1992), que ha sido recuperado junto con otros en el reciente ensayo de Sergio del Molino (2016). El cine de la segunda mitad del siglo XX ha mostrado una imagen negativa de lo rural (González, 1988; Crespo y Quirosa, 2014), cuyos elementos se han distorsionado en la publicidad comercial para atraer a los consumidores (Arranz, 2014). Estos valores que encarnan la naturalidad, la tranquilidad y el aislamiento 
de los espacios rurales influyen en una percepción simbólica de dichos espacios entre la población joven (Pérez y Sánchez, 2007).

La respuesta que intenta romper la separación permanente entre el campo y la ciudad que se ha establecido desde las ciencias sociales (Garayo, 1996) y ha dificultado algunas estrategias de integración entre la escuela y las comunidades rurales (Rodríguez, 2010; Santiago, 2011) proviene de la innovación escolar. En Estados Unidos, el programa PACERS (Program for Academic and Cultural Excellence in Rural Schools) ha trabajado la enseñanza de los espacios rurales de Kansas mediante la relación con contenidos literarios y de la historia del arte a partir del paisaje (San Pedro y López, 2017). El proyecto Nós Propomos! Cidadania e Inovação na Educação Geográfica se ha enfocado hacia la investigación didáctica del alumnado de Educación Secundaria desde la escala local para abordar problemáticas sociales y ambientales relevantes para sus localidades mediante el aprendizaje-servicio (Claudino et al. 2019). Siguiendo en el ámbito iberoamericano, los Proyectos de Extensión de Cátedra de Didáctica de la Geografía de la Universidad del Litoral de Argentina (D’Angelo y Lossio, 2011) han otorgado especial relevancia a la investigación con técnicas cualitativas diversas que han permitido abordar problemas de producción y comercialización de los trabajadores rurales. En el caso español, los especialistas de los proyectos de la Cátedra de Cultura Territorial Valenciana han elaborado materiales didácticos para favorecer la comprensión integral del medio y empoderar a la ciudadanía en la toma de decisiones sobre el futuro de los espacios rurales (Peiró, Álvaro, Vílchez y Farinós, 2019). Como aspectos comunes, en estos proyectos de innovación didáctica se pretende cuestionar la percepción que reduce los atributos rurales con lo agrario (Dirven, 2011) en base a la atribución exclusiva del campo con lo rural (Lockie, Lawrence y Cheshire, 2006; Hermi, 2017), pues los límites entre ambos espacios son casi imperceptibles (Vilà y Capel, 1970; Limonad y MonteMór, 2012).

En síntesis, el interés didáctico de la enseñanza de los espacios rurales reside en mostrar al alumnado las perspectivas de cambio que han acarreado los procesos de globalización para la sociedad rural y permitir la reflexión sobre los contrastes y desigualdades en esas comunidades a escala global. Esto puede hacerse desde el concepto paisaje, si lo consideramos como espacio vivido y construido por las generaciones que han precedido las actuaciones desarrolladas en esos contextos espaciales (Santos, 1996; Pérez, Ezkurdia y Bilbao, 2015). Por ello, la utilización de métodos mixtos desde las representaciones sociales y su aplicación al ámbito de la didáctica de las ciencias sociales permiten profundizar el diagnóstico sobre las concepciones escolares de estos espacios, como expondremos en el siguiente apartado.

\section{METODOLOGÍA}

La teoría de las representaciones sociales planteada por Moscovici (1978) permite conocer cómo las personas reproducen el contenido de la ciencia para obtener un conocimiento útil y con sentido para interpretar el mundo (Moscovici y Hewstone, 1986). En nuestro caso supone analizar la forma en la que el alumnado de Educación Secundaria y Bachillerato forma un pensamiento sobre los espacios rurales a partir de la asimilación y transformación de saberes que les llegan desde diferentes fuentes y contextos de información (Jovchelovitch, 2008). Aquí es donde las representaciones sociales (en sentido teórico y metodológico) deben diferenciarse de algunos conceptos (percepción, inferencia, ideología...) para acceder a sus dimensiones (simbólicas, culturales y prácticas) de los fenómenos sociales (Jodelet, 2009).

La percepción designa un proceso fisiológico que ha sido aplicado a estudios geográficos, en concreto, desde la Geografía de la Percepción y del Comportamiento, a partir de la relación entre Psicología y Geografía (Capel, 1973). El comportamiento ha sido estudiado desde las representaciones sociales (Sammut et al. 2015) puesto que se refleja en el espacio y puede analizarse desde dicha escuela de pensamiento geográfico a partir de la adaptación de la trialéctica de Soja (2008) propuesta por Souto (2018) para la didáctica de la geografía en base a presupuestos socioconstructivistas (Vigotsky, 1993). En la práctica, la representación de los espacios se filtra a partir de las percepciones o espacio percibido de la Geografía de la Percepción y del Comportamiento, como se ha analizado para el caso de la comprensión de los espacios rurales en Chile (Araya, Souto y Herrera, 2015).

En el caso que nos ocupa los sujetos son 581 estudiantes de tercer curso de Educación Secundaria 
(393 estudiantes) y de segundo de Bachillerato (188) de la provincia de Valencia (Figura 1) que fueron seleccionados mediante un muestreo no probabilísticointencional (Tabla 1), que se ajusta a criterios espaciales y didácticos. En el primer caso se ha tenido en cuenta el contexto geográfico del municipio, de acuerdo a la clasificación tripartita (sistema rural, franja intermedia del territorio y cota 100) que se establece en la Estrategia Territorial de la Comunidad Valenciana 2030. Así, los municipios rurales (Villar del Arzobispo, Requena, Càrcer y Villalonga de la Safor) han quedado enmarcados en el sistema rural; a la franja intermedia pertenecen los municipios periurbanos (Riba-roja de Turia, Torrent y Paiporta), mientras que a la cota 100 se corresponde con los sistemas urbanos que se han equiparado a los tres centros escolares ubicados en la ciudad de Valencia (Benimaclet, Eixample y Poblados Marítimos). En el segundo caso, se ha considerado que los centros escolares tuviesen algún proyecto de innovación educativa sobre la enseñanza de los espacios rurales o temáticas afines, tal y como ha ocurrido en varios centros de la ciudad de Valencia.

Figura 1. Localización de los municipios que han participado con centros escolares en el contexto de la clasificación espacial de la Estrategia Territorial Valenciana 2030.
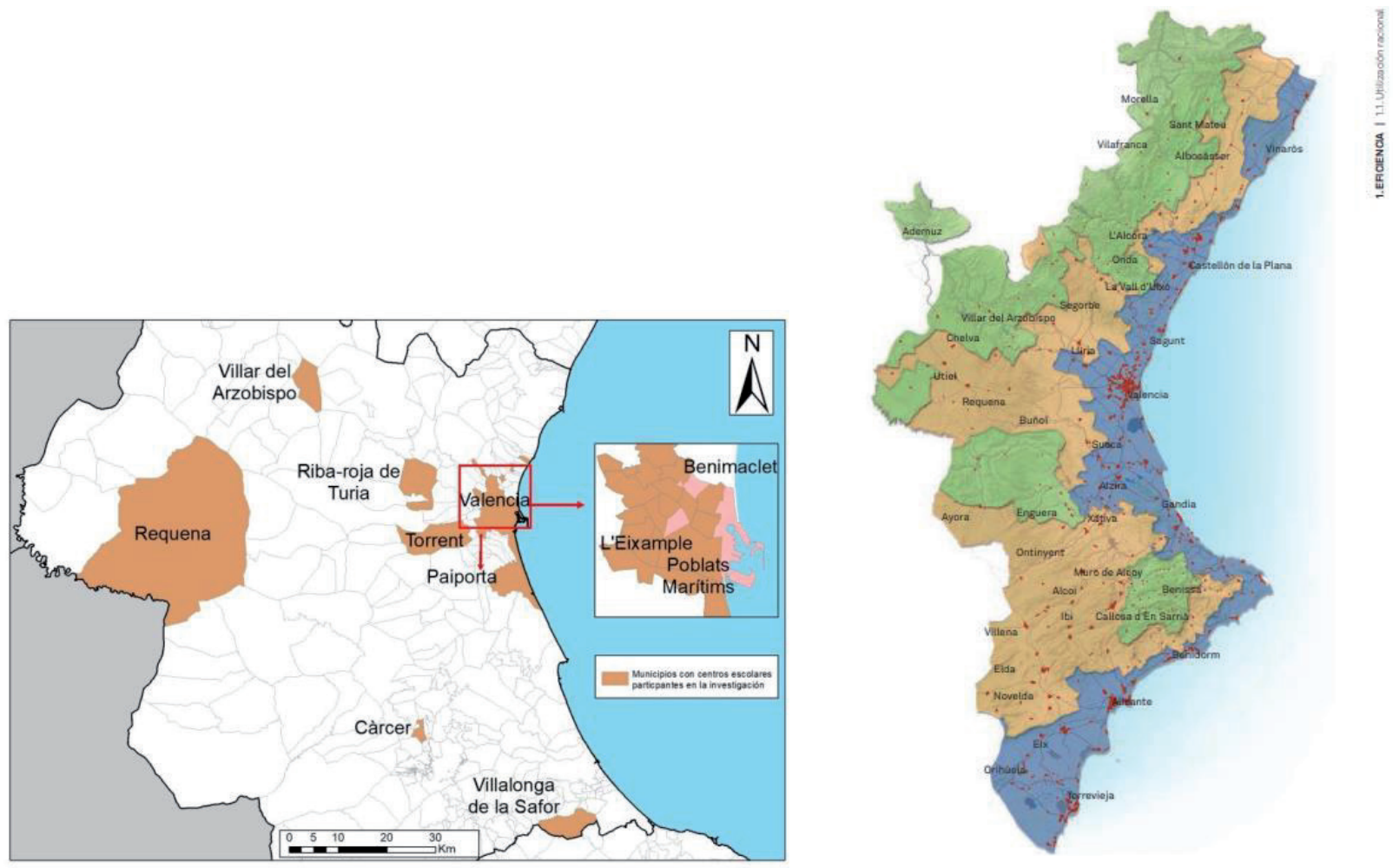

Fuente: Elaboración propia y Estrategia Territorial Valenciana 2030. El color verde pertenece al sistema rural, el marrón a la franja intermedia y el azul a la cota 100

Con esta base teórico-metodológica, la propuesta de Banchs (2000) puede ser aplicada al estudio de las representaciones escolares de los espacios rurales por parte del alumnado. Por un lado, se utilizará el enfoque estructural para conocer los elementos que organizan la representación social, que serán analizados mediante el software Evocation 2005, siguiendo los presupuestos teóricos de Abric (2001). Por otro lado, se recurrirá al enfoque procesual para dotar de significado a esa estructura representacional mediante la interpretación de los dibujos del alumnado, entrevistas semiestructuradas y la discusión con estudios similares. En suma, la combinación de ambos enfoques nos hace decantarnos por un método exploratorio secuencial mixto (Creswell, 2014), que suple la falta de métodos propios de la teoría de las representaciones sociales (Moscovici, 2019). 
Tabla 1. Muestra de alumnado por curso y municipio participante en esta investigación.

\begin{tabular}{lc|c|c}
\multicolumn{1}{c}{ Localidad } & $3^{\circ}$ ESO & $\begin{array}{c}2^{\circ} \text { de } \\
\text { Bachillerato }\end{array}$ & Total \\
\hline Torrent & 24 & - & 24 \\
\hline Benimaclet & - & 27 & 27 \\
\hline $\begin{array}{l}\text { Villar del } \\
\text { Arzobispo }\end{array}$ & 36 & 14 & 50 \\
\hline Requena & 66 & 20 & 86 \\
\hline $\begin{array}{l}\text { Valencia } \\
\text { (Eixample) }\end{array}$ & 24 & 29 & 23 \\
\hline Càrcer & 15 & 8 & 85 \\
\hline $\begin{array}{l}\text { Villalonga } \\
\text { de la Safor }\end{array}$ & 69 & 16 & 79 \\
\hline Riba-roja de Túria & 67 & 12 & 120 \\
\hline Paiporta & 69 & 51 & 34 \\
\hline $\begin{array}{l}\text { Valencia (Poblats } \\
\text { Marítims) }\end{array}$ & 23 & 11 & 581 \\
\hline Total & 393 & 188 & $(100 \%)$ \\
\hline
\end{tabular}

Fuente: Elaboración propia.

De acuerdo a lo anterior, lo que se pretende con esta investigación es lo siguiente: conocer los elementos que inciden en la representación que tiene el alumnado de la provincia de Valencia sobre los espacios rurales, pues el contexto puede influir en la cosmovisión que tienen sobre esos espacios en función de su consideración como espacios vividos, percibidos o concebidos, con un grado diferente de idealización. Por ello, se ha elaborado el siguiente sistema de categorías (Tabla 2) donde se especifica cómo se va a medir la influencia del contexto en la formación de la representación sobre los espacios rurales.

Tabla 2. Sistemas de categorías que guían la investigación.

\begin{tabular}{ll}
$\begin{array}{l}\text { Preguntas metodológicas de } \\
\text { investigación }\end{array}$ & \multicolumn{1}{c}{ Sistemas de categorías } \\
$\begin{array}{l}\text { ¿Cómo influye la } \\
\text { representación del espacio } \\
\text { en la concepción escolar } \\
\text { del medio rural? }\end{array}$ & $\begin{array}{l}\text { Categoría 1: La representación social } \\
\text { del medio rural está condicionada } \\
\text { por el espacio concebido. Nivel } \\
\text { máximo de idealización. }\end{array}$ \\
\hline $\begin{array}{l}\text { ¿Cómo influye la práctica } \\
\text { social en la concepción } \\
\text { escolar del medio rural? }\end{array}$ & $\begin{array}{l}\text { Categoría 2: La representación social } \\
\text { del medio rural está condicionada } \\
\text { por el espacio percibido. Nivel }\end{array}$ \\
intermedio de idealización.
\end{tabular}

Fuente: Elaboración propia a partir de la interpretación de Soja (2008) adaptada por Souto (2018) a la didáctica de la geografía.
Como esos elementos que se integran en el sistema de categorías son parte de una estructura con un significado propio es necesario utilizar los dos enfoques metodológicos propios de las representaciones sociales, como se expondrá a continuación.

\subsection{El enfoque estructural: cuestionario de asociación libre de palabras y análisis cuantitativo de la figura humana en las representaciones pictóricas y en los libros de texto}

El alumnado participante (581 estudiantes: 393 de $3^{\circ}$ ESO y 188 de $2^{\circ}$ de Bachillerato) de diez centros escolares de la provincia de Valencia respondió un cuestionario mixto, cuya primera parte (tres primeras cuestiones) es un test de asociación libre de palabras (Agüero y Chama, 2009; Navia y Estrada, 2012). En la primera pregunta el alumnado escribe cinco palabras que asocia al concepto medio rural, que después ordena de mayor a menor importancia (escala de 1 a 5) en la segunda pregunta y posteriormente define la palabra que considera más importante (valor 1) en la tercera cuestión. Los datos de la segunda pregunta son analizados por el software Evocation 2005 (previa tabulación en un archivo CSV), que ha sido utilizado en otras investigaciones (Lopes, 2010; Silva y Viveiros, 2017) bajo las directrices de la teoría de las representaciones sociales y su aplicación a la educación (Domingos, 2000; Saraiva, 2007), en consonancia con los estudios del contenido representacional del Grupo Midi (Flament, 2001). En la cuarta pregunta de ese mismo cuestionario se pidió al alumnado que dibujase lo que entiende por medio rural en un espacio acotado para tal fin y marcase tres emociones de entre diez proporcionadas, que seguían el modelo de Ekman y Friesen (Martínez, 1991). Estos dibujos son semejantes a los esquemas mentales (Kosslyn, 1981) y se han utilizado en la Geografía de la Percepción y del Comportamiento como técnica complementaria a los cuestionarios (Vara, 2010). Desde esta vertiente de análisis nos ha interesado el recuento de los dibujos en los que ha aparecido la figura humana (Tabla 3), un aspecto que hemos comparado en ambos cursos a partir de la aplicación del test chi-cuadrado. 
Tabla 3. Recuento de las representaciones pictóricas del alumnado en ambos cursos.

\begin{tabular}{ll|c|c|c}
\multirow{2}{*}{ Presencia humana } & \multicolumn{2}{|c|}{ Representaciones pictóricas } & \\
\cline { 3 - 4 } & $\begin{array}{c}\text { Sin presencia } \\
\text { humana }\end{array}$ & & Total \\
\hline \multirow{2}{*}{ Curso } & $3^{\circ} \mathrm{ESO}$ & 42 & 351 & 393 \\
\cline { 2 - 4 } & $2^{\circ} \mathrm{BACH}$ & 29 & 158 & 187 \\
\hline Total & & 71 & 509 & 580 \\
\hline
\end{tabular}

Fuente: Eaboración propia.

Para complementar la representación del alumnado con otras fuentes se procedió al recuento de las fotografías que aparecen en veintiocho libros de texto (dieciséis en $3^{\circ}$ ESO y doce en $2^{\circ}$ de Bachillerato) entre 1959 y 2016 (Tabla 4), que fueron seleccionados de acuerdo a la ley educativa, la editorial y el curso correspondiente a la muestra del alumnado. La mayoría son libros de texto de las cinco editoriales (Anaya, Santillana, SM, Vicens Vives y Ecir) cuya presencia en los centros escolares ronda el 80\% (Valls, 2012). De modo similar a lo enunciado para los dibujos del alumnado, se ha aplicado la prueba chi-cuadrado para analizar la presencia de la figura humana en las fotografías de los libros de texto para ambos cursos.

Tabla 4. Recuento de las fotografías de los libros de texto en ambos cursos.

\begin{tabular}{|c|c|c|c|c|}
\hline \multirow{2}{*}{\multicolumn{2}{|c|}{$\begin{array}{c}\text { Presencia de la figura } \\
\text { humana }\end{array}$}} & \multicolumn{2}{|c|}{ Fotografías } & \multirow[b]{2}{*}{ Total } \\
\hline & & $\begin{array}{l}\text { Sin presencia de } \\
\text { la figura humana }\end{array}$ & & \\
\hline \multirow[t]{2}{*}{ Curso } & $3^{\circ} \mathrm{ESO}$ & 137 & 280 & 417 \\
\hline & $2^{\circ}$ Bachillerato & 77 & 122 & 199 \\
\hline Total & & 214 & 402 & 616 \\
\hline
\end{tabular}

Fuente: Elaboración propia.

2.2. El enfoque procesual: la comprensión del significado de la representación social

Los primeros datos analizados desde esta vertiente han sido los significados de las palabras del núcleo central que el alumnado ha explicado en la tercera pregunta del cuestionario. Para ello se han establecido categorías a partir del programa Atlas ti (versión 7.5.4.) en las que se ha obtenido el significado y las relaciones entre las palabras que estructuran el contenido representacional. De modo similar, se ha interpretado el significado de los dibujos del alumnado en base a tres categorías (paisaje ecológico, paisaje cultivado y paisaje residencial), que siguen las funciones de los espacios rurales (Hudault, 2011) en términos de paisaje (Cavalcanti, 2014) y la división tripartita del paisaje de Ortega (2000). En esa yuxtaposición de funciones y elementos se han superpuesto tres niveles de representación (idealización, práctica social y presencia humana) a partir de la interpretación de Morales (2012).

En cuanto a los libros de texto se ha realizado un análisis crítico del contenido (Fernández, 2005) de los capítulos dedicados a los espacios rurales a partir de un guion elaborado desde algunas investigaciones en didáctica de las ciencias sociales (Souto y Ramírez, 2002; Valls, 2008). En concreto, se ha atendido a las definiciones de los conceptos estructurantes (espacio rural, paisaje rural, poblamiento y política rural) y al nivel cognitivo de las actividades mediante el modelo utilizado en otras publicaciones (Sáiz y Colomer, 2014). Los contenidos estudiados y las fuentes de información para documentarse acerca de los espacios rurales han sido dos aspectos sobre los que se les ha preguntado a los 58 estudiantes que han participado en entrevistas semiestructuradas o en grupos de discusión, dependiendo de la disponibilidad del tiempo escolar. No obstante, la entrevista semiestructurada dota de menor libertad de acción que los grupos de discusión (Butler, 1996), que permiten captar mejor la información y su interrelación entre los participantes (Freitas et al. 1998; Sagoe, 2012). En ambos casos, se ha utilizado un sistema de transcripción que ha tenido en cuenta los aspectos prosódicos y los cambios de ideas de los participantes (Onwuegbuzie et al. 2009) para analizar sus respuestas en base al esquema de trabajo de otras investigaciones (García Calvente y Mateo Rodríguez, 2000; García-Ruiz y Lena-Acebo, 2019).

Finalmente y debido a la diversidad de los datos obtenidos en ambos enfoques se procedió a la triangulación (Flick, 2004; Okuda-Benavides y GómezRestrepo, 2005) para poder ofrecer una interpretación conjunta de la estructura y del significado de los elementos que intervienen en la representación de los espacios rurales. Así es como se van a presentar los resultados principales y su discusión complementaria.

\section{RESULTADOS Y DISCUSIONES}

La estructura del contenido representacional que ha proporcionado el software Evocation 2005 permite 
diferenciar entre un núcleo duro (conceptos animales y campo) y los elementos periféricos que concretan el significado de dicho núcleo (Tabla 5). Campo es el concepto que mejor concentra el valor simbólico y el poder asociativo (Sá, 2002) de lo que el alumnado entiende por espacio rural, como se ha comprobado en la categorización de las definiciones de los conceptos más valorados en la pregunta tercera del cuestionario y en las entrevistas del alumnado (Tabla 6). El campo es el contenido de lo rural, que se asocia tanto a la naturaleza como a las actividades agrarias (contenido descriptivo) en un contexto de tranquilidad (contenido prescriptivo).

Tabla 5. Estructura del contenido representacional de espacio rural

\begin{tabular}{|c|c|c|c|c|c|}
\hline \multicolumn{3}{|c|}{$\mathrm{F} \geq 131$ y Rango $<2,5$} & \multicolumn{3}{|c|}{$F \geq 131$ y Rango $\geq 2,5$} \\
\hline \multicolumn{2}{|c|}{ Frecuencia } & Rango & \multicolumn{2}{|c|}{ Frecuencia } & Rango \\
\hline Animales & 202 & 2,383 & Vegetación & 277 & 2,509 \\
\hline Campo & 162 & 2,309 & & & \\
\hline \multicolumn{3}{|c|}{ (Núcleo central) } & \multicolumn{3}{|c|}{ (Elementos intermedios) } \\
\hline \multicolumn{3}{|c|}{$\mathrm{F} \leq 131$ y Rango $<2,5$} & \multicolumn{3}{|c|}{$\mathrm{F} \leq 131$ y Rango $>2,5$} \\
\hline \multicolumn{2}{|c|}{ Frecuencia } & Rango & \multicolumn{2}{|c|}{ Frecuencia } & Rango \\
\hline Naturaleza & 96 & 1,979 & Montañas & 119 & 2,639 \\
\hline Pueblos & 92 & 2,272 & Casa-rural & 107 & 2,794 \\
\hline Tranquilidad & 87 & 2,230 & Río & 78 & 2,628 \\
\hline Agricultura & 87 & 2,391 & & & \\
\hline \multicolumn{3}{|c|}{ (Elementos periféricos) } & \multicolumn{3}{|c|}{ (Elementos periféricos) } \\
\hline
\end{tabular}

Fuente: Elaboración propia a partir de los resultados del software Evocation 2005.
Campo denota ser un concepto prominente en la estructura representacional y alberga el contenido descriptivo al asociarse con animales y vegetación, además de con los elementos intermedios (naturaleza, pueblos y agricultura). Es un término más prominente que animales, pues sus significados como área natural y de cultivo con connotaciones de tranquilidad se vinculan con las definiciones del mismo concepto en los diccionarios históricos de la Real Academia de la Lengua Española. Además se relaciona con el concepto country o espacio que no incluye la sociedad, por contraposición a the country (Williams, 2001), esto es, una cosmovisión similar a la de parque natural por el alumnado de Educación Primaria, en la que el núcleo duro alude a un espacio de animales y plantas (Santana, 2019).

En las representaciones pictóricas del alumnado se ha constatado el predominio de los valores naturales de los paisajes rurales (Figura 2). El 47.8\% de los dibujos representan la función ecológica en $3^{\circ} \mathrm{ESO}$, especialmente entre el alumnado de áreas periurbanas. En cambio, el alumnado de áreas rurales otorga más importancia a la función económica en ambos cursos y en sus explicaciones mencionan los cultivos de sus localidades.

Tabla 6. Aspectos comunes en las definiciones escolares del alumnado con su frecuencia.

\begin{tabular}{|c|c|c|c|c|c|c|}
\hline & Animales & Fc & $\mathrm{Fe}$ & Campo & $\mathrm{Fc}$ & $\mathrm{Fe}$ \\
\hline Naturaleza & $\begin{array}{l}\text { El medio rural es natural } \\
\text { por definición y contiene } \\
\text { diversas especies de } \\
\text { animales que no pueden } \\
\text { verse en otros espacios. }\end{array}$ & 21 & 7 & $\begin{array}{l}\text { Porque el medio rural } \\
\text { son campos rodeados } \\
\text { de zonas naturales. }\end{array}$ & 29 & 10 \\
\hline Agricultura & $\begin{array}{l}\text { En lo rural se encuentran } \\
\text { animales (vacas, ovejas...) } \\
\text { que complementan la } \\
\text { actividad agraria. }\end{array}$ & 16 & 5 & $\begin{array}{l}\text { Asocio las actividades } \\
\text { agrícolas a los campos } \\
\text { del interior de España. }\end{array}$ & 23 & 8 \\
\hline Tranquilidad & $\begin{array}{l}\text { Cuando vas a un espacio rural } \\
\text { escuchas el sonido de algunos } \\
\text { animales y me da tranquilidad. }\end{array}$ & 12 & 2 & $\begin{array}{l}\text { En el campo no } \\
\text { vives el estrés de la } \\
\text { ciudad y se disfruta } \\
\text { de más tranquilidad, } \\
\text { paz y sosiego. }\end{array}$ & 18 & 4 \\
\hline
\end{tabular}

Fuente: Elaboración propia a partir de la categorización mediante Atlas.ti (versión 7.5.4.). para la tercera pregunta del cuestionario (Fc) y la primera pregunta de las entrevistas semiestructuradas (Fe). Los números indican el número de casos similares registrados en ambos casos. 
Figura 2. Clasificación de las representaciones pictóricas del alumnado por su procedencia geográfica.

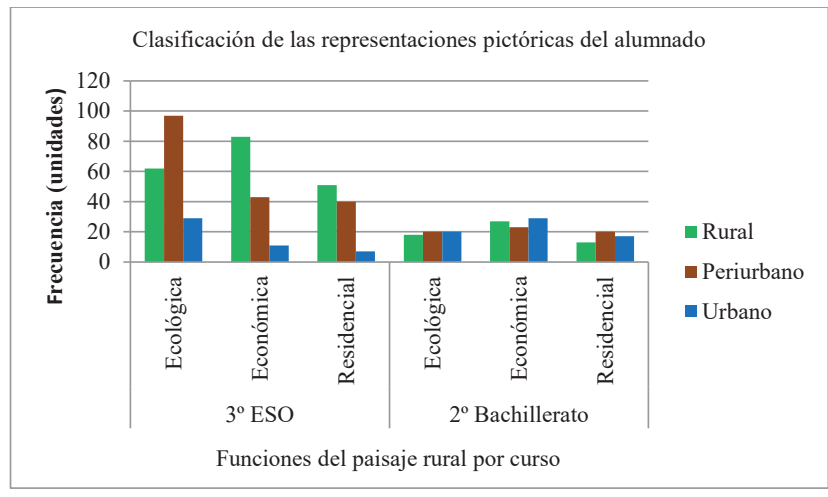

Fuente: Elaboración propia a partir de los dibujos del alumnado.

A las representaciones pictóricas han asociado una serie de emociones. En $3^{\circ}$ ESO las 1.039 emociones recopiladas siguen una secuencia mayoritaria de alegría-serenidad-admiración, mientras que las 453 de segundo de Bachillerato son serenidad-alegríamelancolía (Figura 3). En conjunto, el 74\% de las secuencias emocionales coinciden en dotar a los espacios rurales de emociones positivas (alegría y serenidad) con la particularidad de la melancolía (tercera emoción en la secuencia proporcionada en el cuestionario) para $2^{\circ}$ de Bachillerato, por cuanto a este alumnado se le infiere más madurez intelectual y experiencia en el conocimiento de estos espacios. Según la procedencia geográfica del alumnado, la estructura genérica se rompe en el caso de las áreas rurales porque este alumnado concede más importancia a la admiración, pues valora estos espacios por residir en ellos. En las localidades urbanas y periurbanas añade la melancolía, lo que se interpreta por el valor de tranquilidad comprobado en la estructura representacional. Esta concepción positiva puede dificultar la concienciación de la población escolar por las problemáticas de los espacios rurales, como han señalado otras investigaciones (Pérez y Sánchez, 2007; Capdevila, 2017), aunque esta tendencia se reduce con el alumnado procedente de áreas rurales (Caurín, Morales y Solaz, 2012; Araya, Souto y Herrera, 2015).
Figura 3. Recuento de las emociones asociadas a las representaciones pictóricas del alumnado.

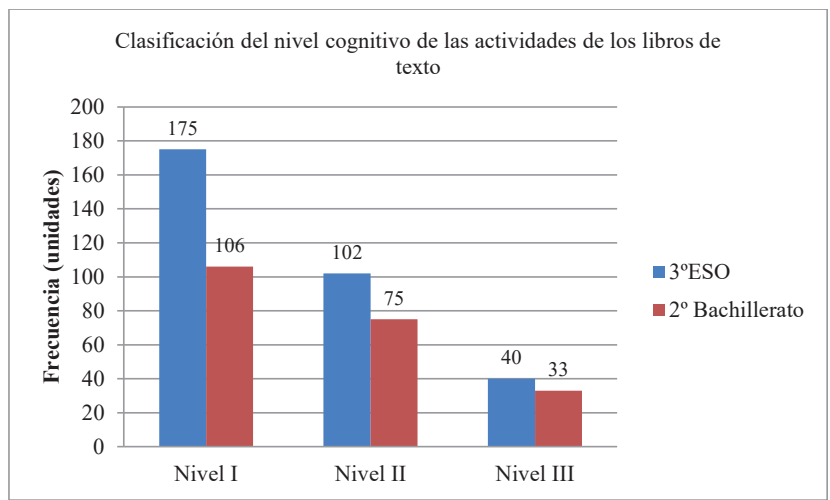

Fuente: Elaboración propia a partir de un recuento individualizado.

El siguiente aspecto que nos aboca hacia la representación idealizada de los espacios rurales es el análisis de la presencia humana. Se ha procedido a la aplicación de la prueba estadística chi-cuadrado para comparar la significación entre los dibujos del alumnado que han representado la figura humana para ambos cursos (Tabla 7). El resultado para las 580 representaciones pictóricas (un alumno no la realizó) es 0,98 (p-valor) que por ser superar 0,05 (valor alpha) permite afirmar que existe una tendencia similar a prescindir de la figura humana en los dibujos del alumnado tanto en $3^{\circ}$ ESO como en $2^{\circ}$ de Bachillerato. En la figura 4 se han seleccionado tres dibujos, uno para cada categoría (paisaje ecológico, paisaje económico y paisaje residencial) que manifiestan una tendencia a representar mayoritariamente los elementos naturales y de forma secundaria la actividad económica, en la que apenas se intuye la presencia humana, en consonancia con las representaciones pictóricas escolares de paisaje de otras investigaciones (Liceras, 2003 y García de la Vega, 2014). Esta añoranza por el medio ambiente rural es coincidente con la tendencia que se difundió en la pintura renacentista en la que la representación humana era secundaria, pues la vida en el campo era anhelada por la burguesía (Maderuelo, 1996). 
Tabla 7. Resultados de la prueba chi-cuadrado aplicada a las representaciones pictóricas del alumnado.

\begin{tabular}{l|ccc|c|c} 
& Valor & gl & $\begin{array}{c}\text { Sig. } \\
\text { asintótica } \\
(2 \text { caras })\end{array}$ & $\begin{array}{c}\text { Sig. exacta } \\
(2 \text { caras })\end{array}$ & $\begin{array}{c}\text { Sig. exacta } \\
\text { (1 cara })\end{array}$ \\
$\begin{array}{l}\text { Chi-cuadrado } \\
\text { de Pearson }\end{array}$ & 2,741 & 1 &, 098 & & \\
\hline $\begin{array}{l}\text { Corrección de } \\
\text { continuidad }\end{array}$ & 2,311 & 1 &, 128 & & \\
\hline $\begin{array}{l}\text { Razón de } \\
\text { verosimilitud }\end{array}$ & 2,650 & 1 &, 104 & \\
\hline $\begin{array}{l}\text { Prueba exacta } \\
\text { de Fisher }\end{array}$ & & & & & \\
\hline $\begin{array}{l}\text { Asociación } \\
\text { lineal por lineal }\end{array}$ & 2,737 & 1 &, 098 & & \\
\hline $\begin{array}{l}\text { N de casos } \\
\text { válidos }\end{array}$ & 580 & & & & \\
\hline
\end{tabular}

Fuente: Elaboración propia a partir del programa IBM SPSS Statics 22.
El análisis del contenido de los libros de texto refleja que los espacios rurales aparecen separados de los capítulos dedicados a los espacios urbanos en la totalidad de las obras consultadas. Los contenidos denotan la imagen de espacios agrarios, con escasas referencias a la multifuncionalidad y un relato que se centra en la descripción de la morfología del paisaje y el análisis de las políticas sobre los territorios rurales. Así se manifiesta en la reiteración de los conceptos hábitat y poblamiento, paisaje y política agraria o rural, que son los que más se repiten en la mayoría de los libros de texto analizados. Hábitat y poblamiento se definen indistintamente, pese a que la definición del segundo hace referencia a la distribución de los poblados a lo largo de un territorio (Souto, 1982). El

Figura 4. Modelos de representaciones pictóricas del alumnado.
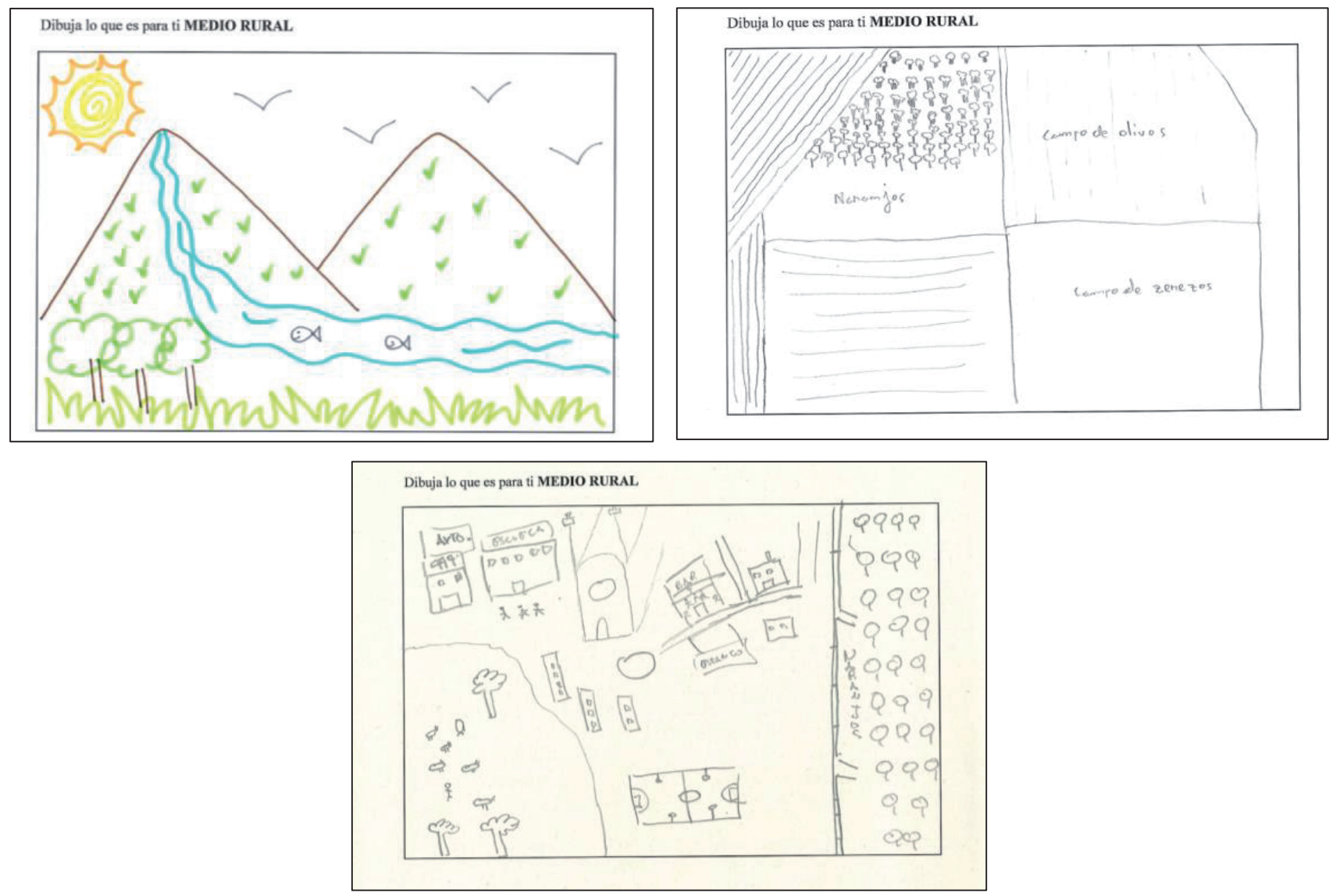

Fuente: elaboración propia a partir de los dibujos del alumnado. El primero de la parte superior izquierda representa la función ecológica, a su derecha la función económica y en la parte inferior la función residencial de los espacios rurales. 
paisaje ha adquirido una connotación natural patente a partir de la década de 1990 cuando ha sustituido al concepto espacio y se separa del estudio de la acción antrópica sobre el territorio, un aspecto que entronca con la escasa presencia de la figura humana en las representaciones pictóricas del alumnado y dificulta la comprensión integral propuesta por Ortega (2000) (Tabla 8). La actividad humana de los espacios rurales queda supeditada casi exclusivamente a las actividades del sector primario, lo que coincide con la visión economicista que han diagnosticado otros autores en los libros de texto en otras regiones españolas (Armas, Rodríguez y Macía, 2018; Morón y Morón, 2019).

Las actividades propuestas son predominantemente de nivel cognitivo bajo (52.9\%), lo que indica que el alumnado se limita a responder parafraseando el texto o copiando literalmente las definiciones de los principales conceptos, sin diferencias significativas entre ambos cursos (Figura 5). A modo de ejemplo la siguiente actividad en $3^{\circ}$ ESO: ¿Qué tipo de paisaje agrario es característico del Mediterráneo? ¿Qué características tiene? (Méndez, Gutiérrez y Guerra, 2010: 83) y otra en $2^{\circ}$ de Bachillerato: Lee de nuevo el epígrafe sobre los factores políticos y resume cuáles eran los factores de la PAC en un primer período (Abascal et al. 2009: 281). La diferencia en los dos siguientes niveles es menor entre ambos cursos, aunque generalmente el mayor número de actividades de bajo nivel cognitivo también se ha registrado para el caso de los libros de texto de historia en Educación Secundaria y Bachillerato (Sáiz, 2011; Martínez y Gómez, 2018).

Figura 5. Nivel cognitivo de las actividades de los libros de texto en ambos cursos.

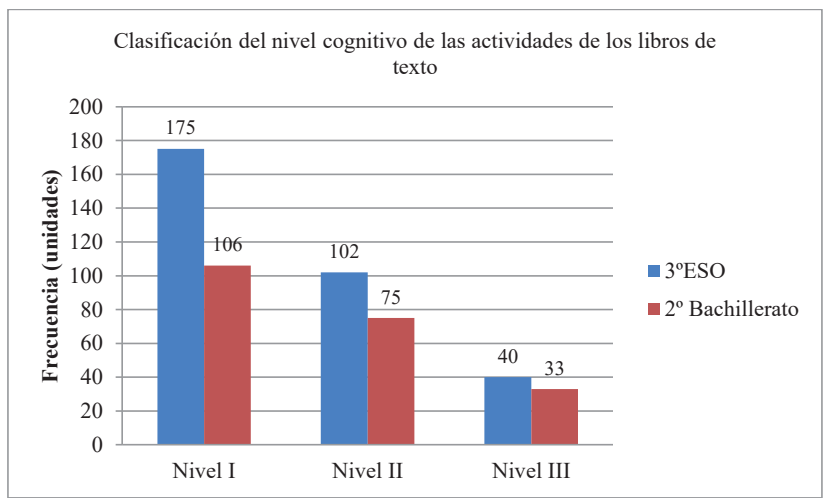

Fuente: Elaboración propia.

De modo similar a lo que se ha analizado para las representaciones pictóricas del alumnado se ha aplicado la prueba chi-cuadrado a las 616 fotografías de los libros de texto en ambos cursos (Tabla 9). El resultado es 0,155 (valor superior a alpha: 0,05 ) lo que indica que la diferencia entre las fotografías con presencia humana en los libros de texto no es significativa para $3^{\circ} \mathrm{ESO}$ y $2^{\circ}$ de Bachillerato.

Tabla 8. Síntesis de algunas definiciones de los conceptos estructurantes sobre los espacios rurales.

\begin{tabular}{|c|c|c|}
\hline & $3^{\circ} \mathrm{ESO}$ & $2^{\circ}$ de Bachillerato \\
\hline Hábitat y/o poblamiento & $\begin{array}{l}\text { El hábitat. La casa rural es uno de los elementos } \\
\text { representativos del paisaje agrario (...). (Gorgues, } \\
\text { Argente y Tolosa, 2007: 17). }\end{array}$ & $\begin{array}{l}\text { "Hábitat" (...) se refiere al modo como suele el hombre } \\
\text { construir su vivienda en los distintos lugares de la tierra } \\
\text { (...). (García-Manrique, 1959: 64). }\end{array}$ \\
\hline Paisaje agrario o rural & $\begin{array}{l}\text { (...) paisaje agrario (..) modificado con la finalidad } \\
\text { de obtener productos de la naturaleza y que ha ido } \\
\text { evolucionando para aumentar su aprovechamiento (Albet } \\
\text { et al. 2008: } 60 \text { ). }\end{array}$ & $\begin{array}{l}\text { El paisaje agrario es el resultado de las diversas relaciones } \\
\text { entre el ser humano y el medio físico (García et al.2009: } \\
74 \text { ). }\end{array}$ \\
\hline Política agraria o rural & $\begin{array}{l}\text { Las medidas agrarias puestas en marcha por los gobiernos } \\
\text { también influyen sobre las políticas agrarias. Las más } \\
\text { habituales son las reformas agrarias para conseguir un } \\
\text { reparto más equilibrado de la propiedad (...). (Burgos y } \\
\text { Muñoz-Delgado, 2015:42) }\end{array}$ & $\begin{array}{l}\text { (...) la Política Agraria Común sufre una fuerte } \\
\text { transformación en los años ochenta, pues reduce } \\
\text { su porcentaje en el presupuesto comunitario (...). } \\
\text { Igualmente la agricultura se plantea como parte integrante } \\
\text { del desarrollo rural en su conjunto, potenciando las } \\
\text { medidas agro-ambientales y el paisaje forestal (Souto, } \\
\text { 2006: 50). }\end{array}$ \\
\hline
\end{tabular}

Fuente: Elaboración propia a partir del análisis de los libros de texto (1959-2016). 
Tabla 9. Resultados de la prueba chi-cuadrado aplicada a las fotografías de los libros de texto.

\begin{tabular}{l|cc|c|c|c} 
& Valor & gl & $\begin{array}{c}\text { Sig. } \\
\text { asintótica } \\
\text { (2 caras) }\end{array}$ & $\begin{array}{c}\text { Sig. exacta } \\
(2 \text { caras })\end{array}$ & $\begin{array}{c}\text { Sig. exacta } \\
\text { (1 cara) }\end{array}$ \\
$\begin{array}{l}\text { Chi-cuadrado } \\
\text { de Pearson }\end{array}$ & 2,026 & 1 &, 155 & & \\
\hline $\begin{array}{l}\text { Corrección de } \\
\text { continuidad }\end{array}$ & 1,777 & 1 &, 183 & \\
\hline $\begin{array}{l}\text { Razón de } \\
\text { verosimilitud }\end{array}$ & 2,010 & 1 &, 156 & \\
\hline $\begin{array}{l}\text { Prueba exacta } \\
\text { de Fisher }\end{array}$ & & & & & \\
\hline $\begin{array}{l}\text { Asociación } \\
\text { lineal por lineal }\end{array}$ & 2,023 & 1 &, 155 & \\
\hline $\begin{array}{l}\text { N de casos } \\
\text { válidos }\end{array}$ & 616 & & & \\
\hline
\end{tabular}

Fuente: Elaboración propia a partir del programa IBM SPSS Statics 22.

La escasa consideración de la presencia humana en las fotografías de los libros de texto puede inducir en las representaciones pictóricas del alumnado, pues su uso con carácter envolvente (Parcerisa, 1996; Merchán, 2011; Martínez-Valcárcel, 2016) ronda entre el 50\% del profesorado de Educación Secundaria de Matemáticas y el $80 \%$ en ciencias sociales, especialmente entre las editoriales que controlan el mercado internacional (Murillo, 2003; Valls, 2012). Las fotografías muestran los campos cultivados con algunas referencias a la mecanización y el trabajo de personas inmigrantes, con muy poca presencia de la mujer. Estos rasgos son coincidentes con los analizados en los libros de texto de Ensino Medio en Brasil, aunque en este país los contenidos rurales son menos descriptivos y no van separados de los capítulos dedicados a los espacios urbanos (García-Monteagudo, 2019).

A grandes rasgos ese carácter descriptivo de los textos escolares se une a la tendencia conservadora de esos contenidos (Tonini, Claudino y Souto, 2015), lo que no favorece la comprensión de los problemas sociales y ambientales por parte del alumnado (Vázquez y Aguaded, 2001; Marcén y Molina, 2006). El relato de los libros de texto tiene menor importancia entre el alumnado respecto de otras fuentes de información. El $80 \%$ de alumnado entrevistado entiende los espacios rurales a partir de la percepción de otras fuentes, especialmente la televisión, internet y las redes sociales. La publicidad reproduce estereotipos de los espacios rurales profundos (Sancho y Reinoso, 2012; Molinero, 2019), con alusiones a la vestimenta, el lenguaje y la alimentación (Arranz, 2014), que han sido tomados de valores del cine rural de la década de 1970 (González, 1988; Crespo y Quirosa, 2014). Otras investigaciones han demostrado que la tendencia a representar el medio natural de forma idealizada, como ocurre en el caso del espacio rural, es común entre el alumnado de zonas rurales y urbanas (Arcury y Howard, 1993; Matthews et al. 2000; Paniagua y Hoggart, 2002), por lo que es importante que con los resultados que ofrecemos se contribuya a una enseñanza lo más ajustada posible a la realidad multifuncional de estos espacios en la actualidad.

\section{CONCLUSIONES}

Esta investigación constituye un inicio en el conocimiento de los espacios rurales por parte de la población escolar desde la confluencia entre la teoría de las representaciones sociales y la vertiente humanista de la geografía de la percepción y del comportamiento. La dimensión espacial tripartita (espacio vivido, espacio percibido y espacio concebido) ha permitido formular las preguntas de investigación que son relevantes para conocer los elementos que influyen en la representación de los espacios rurales en el ámbito escolar. La triangulación de los datos de ambos enfoques (estructural y procesual) permite afirmar que existe una cosmovisión idealizada de los espacios rurales entre el alumnado de $3^{\circ}$ ESO y $2^{\circ}$ de Bachillerato de la provincia de Valencia. Los elementos que intervienen son parte de una representación social que incide en la explicación escolar de los espacios rurales a partir del espacio percibido a través de fuentes ajenas a la cultura canónica de los libros de texto. En estos materiales curriculares los espacios rurales se presentan separados de los espacios urbanos y se supeditan a la función de producción agrícola mediante unos contenidos que no favorecen la explicación de problemas sociales y ambientales entre el alumnado. Se trata de espacios rurales concebidos por oposición a la cultura urbana, desde donde se ha legitimado una representación natural de lo rural encarnada en el concepto campo y que se ha ido plasmando en un relato en diferentes fuentes de información, tan solo cuestionadas parcialmente por una parte del alumnado que habita en áreas rurales del interior de la provincia de Valencia. 
Como los resultados son propios de un estudio de caso con carácter intensivo es preciso profundizar en la investigación mediante la inclusión de otros contextos y centros escolares en una escala geográfica más amplia. En este sentido se ha iniciado el foro 25 dentro del Geoforo Iberoamericano sobre Educación, Geografía y Sociedad, en el que se han registrado más de 200 comentarios de profesorado en activo y en formación de distintas universidades iberoamericanas de Brasil, Colombia, Chile, Perú, Portugal y España. Eso nos permitirá profundizar en el diagnóstico de los elementos que inciden en la formación de una representación sobre los espacios rurales e iniciar proyectos de innovación didáctica en la Enseñanza Media para ayudar al alumnado a comprender la complejidad y las oportunidades de vida y laborales en estos espacios, así como la contribución a una ciudadanía crítica y responsable.

\section{REFERENCIAS BIBLIOGRÁFICAS}

Abascal, F., Cabeza, O., Fernández, V., Redondo, A. y Vázquez, Ma .L. (2009). Geografía. $2^{\circ}$ Bachillerato. Madrid: Santillana.

Abric, J. C. (2001). A abordagem estrutural das representações sociais. Em A.S.P. Moreira y D.C. Oliveira (Orgs.), Estudos interdisciplinares de Representações Sociais (pp. 2738). Goiânia: AB.

Agüero, A. y Chama, M. (2009). Arriesgando la palabra: cultura y psicoanálisis. Buenos Aires: Autores de Argentina.

Albet, A., Benejam, P., Casas, M., Comas, P., Oller, M. (2008): Ciencias Sociales, Geografía. $3^{\circ}$ ESO, Demos. Comunitat Valenciana. Alaquàs: Vicens Vives.

Araya, F., Souto, X.M. y Herrera, Y. (2015). El espacio geográfico, una construcción escolar. Un estudio de caso: los alumnos del valle del Limarí (Chile). Scripta Nova, Revista Electrónica de Geografía y Ciencias Sociales, vol. XIX, 503. Recuperado de: http://revistes.ub.edu/index.php/ ScriptaNova/article/view/15108/18311

Arcury, T.A \& Howard, E. (1993). Rural-urban differences in environmental knowledge and actions. Journal of Environmental Education, 25, 19-25. https://doi.org/10.10 80/00958964.1993.9941940

Armas, F.X., Rodríguez, F. y Macía, X.C. (2018). La olvidada geografía rural en el currículo y manuales de la educación secundaria. Revista de Investigación en Didáctica de las Ciencias Sociales, 3, 4-19. http://dehesa.unex.es/ handle/10662/7979

Arranz, A. (2014). Imágenes del medio rural en la publicidad comercial: análisis y propuestas. Trabajo Final de Grado inédito. Segovia: Facultad de Ciencias Sociales, Jurídicas y de la Comunicación. http://uvadoc.uva.es/ handle/10324/6177

Banchs, M. (2000). Aproximaciones procesuales y estructurales al estudio de las representaciones sociales.
Papers on Social Representations. Textes sur représentations sociales, 9, 310-315.

Burgos, M. y Muñoz-Delgado, Ma .C. (2015). Geografía e Historia. $3^{\circ}$ ESO. Madrid: Anaya.

Butler, S. (1996). Child protection or professional selfpreservation by the baby nurses? Public health nurses and child protection in Ireland. Social Science and Medicine, 43 (3), 303-314. https://doi.org/10.1016/02779536(95)00378-9

Capdevila, C. (2017). Jóvenes en el medio rural: Relaciones sociales y expectativas de futuro en la comarca de La Litera (Huesca). Trabajo Final de Grado inédito. Barcelona: Universitat de Barcelona. http://diposit.ub.edu/dspace/ handle/2445/108547

Capel, H. (1973). Percepción del medio y comportamiento geográfico. Revista de Geografía, 7, 58-150.

Caurín, C., Morales, A.J. y Solaz, J.J. (2012). ¿Es posible un cambio de actitudes hacia un modelo de Desarrollo Sostenible? Didáctica de las Ciencias Experimentales y Sociales, 26, 229-245. https://ojs.uv.es/index.php/dces/ article/view/1926

Cavalcanti, L. (2014). Geografia, escola e construção de conhecimientos. Campinas: Papirus Editora.

Cavalcanti. L. (2010). A geografia e a realidade escolar contemporânea: avanços, caminhos, alternativas. Em: Anais do I Seminário Nacional. Currículo em movimientoperspectivas atuais. Belo Horizonte.

Chapuis, R. (1995). La géographie agraire et la géographie rurale. En: A. Bailly (coord.), Les concepts de la Géographie Humaine. París: Masson.

Claudino, S., Souto, X.M., Rodríguez M.A., Bazolli, J., Lenilde de Araújo, R., Gengnagel, C., Mendes, L., Silva, A. (Org.) (2019). Geografia, Educação e Cidadania. Lisboa: ZOE/Centro de Estudos Geográficos, Instituto de Geografia e Ordenamento do Território da Universidade de Lisboa. https://doi.org/10.33787/CEG20190004

Cloke, P. (2006). Conceptualizing Rurality. In: P. Cloke, T. Marsden \& P.H. Mooney (Eds.), The Handbook of Rural Studies (pp. 18-28). London: Sage Publications.

Crespo, J.M. y Quirosa, V. (2014). La visión del medio rural en el cine español de la primera década del siglo XXI. Nuevos valores en tiempos de cambio. Methaodos, Revista de Ciencias Sociales, 2 (2), 286-294. https://doi. org/10.17502/m.rcs.v2i2.58

Creswell, J.W. (2014). Research Design: Qualitative, Quantitative and Mixed Methods Approaches. Research design Qualitative quantitative and mixed methods approaches. London: Sage Publications.

D’Angelo, M.L., y Lossio, O. (2011). Innovar en la enseñanza de geografía rural en la escuela secundaria: propuesta de desarrollo profesional docente. Revista Geográfica de América Central, II Semestre, 1-12. https://www.revistas. una.ac.cr/index.php/geografica/article/view/2733

Dirven, M. (2011). Corta reseña sobre la necesidad de redefinir "rural". En: M. Dirven, R. S. Echeverri, A. Rodríguez, D. Candia, C. Peña, y S. Faiguenbaum (Coords.), Hacia una nueva definición de 'rural' con fines estadísticos en América Latina (pp. 9-11). Santiago de Chile: CEPAL. 
Domingos, M. (2000). Habitus e representações sociais: questões para o estudo de identidades coletivas. Em: A.S.P., Moreira y D.C. Oliveira (Orgs.), Estudos interdisciplinares de representação social (pp. 117-159). Goiânia: AB.

Entrena, F. (1998). Cambios en la construcción social de lo rural. De la autarquía a la globalización. Madrid: Tecnos.

Esparcia, J. (2012). Evolución reciente, situación actual y perspectivas futuras en el desarrollo rural en España y en la UE. Revue Marocaine d'Administration Locale et de Développement, 79, 53 -84.

Espín, M.P., Vega, P. de y Lagos, M. (2016). Teatro lírico español. Ópera, drama lírico y zarzuela grande entre 1868 y 1925. Madrid: Universidad Nacional de Educación a Distancia.

Estébanez, J. (1986). Tendencias en Geografía rural. En: A. García-Ballesteros (coord.), Teoría y práctica de la geografía (225-257). Madrid: Alhambra.

Estrategia Territorial de la Comunidad Valenciana 2030. Disponible en http://politicaterritorial.gva.es/es/ web/planificacion-territorial-e-infraestructura-verde/ estrategia-territorial-de-la-comunitat-valenciana-77496

Fernández, A. (2005). La importancia de ser llamado «libro de texto». Hegemonía y control del currículo en el aula. Buenos Aires: Miño y Dávila Editores.

Flament, C. (2001). Estrutura e dinâmica das representações sociais. Em: D. Jodelet (Org.), As representações sociais. Tradução, Lílian Ulup (pp. 173-186). Rio de Janeiro: EdUERJ.

Flick, U. (2004). Introducción a la Investigación Cualitativa. Madrid: Morata.

Freitas, H., Oliveira, M., Jenkins, M., \& Popjoy, O. (1998). The Focus Group, A Qualitative Research Method. Isrc, (010298), 1-22. https://doi.org/10.1016/j. watres.2013.02.032

Fusi, J.P. (1992). La aparición de los nacionalismos. Revista del Centro de Estudios Constitucionales, 11, 181-194.

Garayo, J.M. (1996). La sociedad rural en el final de siglo. Inguruak. Revista Vasca de Sociología y Ciencia Política, 16, 61-80.

García Calvente, M. M., y Mateo Rodríguez, I. (2000). El grupo focal como técnica de investigación cualitativa en salud: diseño y puesta en práctica. Atención Primaria, 25(3). https://doi.org/10.1016/S0212-6567(00)78485-X

García de la Vega, A. (2014). El pensamiento crítico en el análisis e interpretación de las representaciones sociales del paisaje. En: R. Martínez y E.M M $^{a}$ Tonda (Eds.), Nuevas perspectivas conceptuales y metodológicas para la educación geográfica (pp. 93-108).Murcia: Asociación de Geógrafos Españoles.

García, E., García, A., Rey, F., Hernández, F. y Goberna, J. (2009). Geografía. 20 Bachillerato. Madrid: Ecir.

García, M.D. (1992). Desarrollo y tendencias actuales de la geografía rural (1980-1990), una perspectiva internacional y una agenda de futuro. Agricultura y sociedad, 62, 167-194.

García-Manrique, E. (1959). Geografía Universal. $2^{\circ}$ curso de Bachillerato. Zaragoza: S.I.
García-Monteagudo, D. (2018). Análisis metodológico de las representaciones sociales del medio rural entre estudiantes de Educación Secundaria: un estudio de caso. En: V. Peris, D. Parra y X.M. Souto (coord.), Repensamos la geografía y la historia para la educación democrática (pp. 75-87). Valencia: Nau Llibres.

García-Monteagudo, D. (2019). Tradiciones en la enseñanza del medio rural desde una perspectiva iberoamericana: análisis de los libros de texto de España y Brasil. En: M. J. Hortas, A. Dias y N. de Alba (Eds.), Enseñar y aprender didáctica de las ciencias sociales: la formación del profesorado desde una perspectiva sociocrítica (pp.50-58). Lisboa: Asociación Universitaria del Profesorado de Didáctica de las Ciencias Sociales.

García-Ruiz, Ma.E. y Lena-Acebo, FJ. (2019). Movimiento Fablab: diseño de investigación mediante métodos mixtos. OBETS, Revista de Ciencias Sociales, 14(2), 373406. doi: 10.14198/OBETS2019.14.2.04

Geoforo Iberoamericano sobre Educación, Geografía y Sociedad: http://geoforoforo2.blogspot.com/

George, P. (1973). Los métodos de la Geografía. Barcelona: Oikos-Tau.

Gómez, J. (2008). La mirada del geógrafo sobre el paisaje: del conocimiento a la gestión. En: J. Maderuelo (coord.), Paisaje y Territorio (pp. 11-56). Madrid: Fundación Beulas, Centro de Arte y Naturaleza (CDAN).

Gómez, J. (2013). Del patrimonio paisaje a los paisajes patrimonio. Documents d'Anàlisis Geogràfica, 59 (1), 5-20.

González, J. (1988). El campo en el cine español. Madrid: Banco de Crédito Agrícola.

Gorgues, R., Argente, Ma .J. y Tolosa, C. (2007). Geografía. $3^{\circ}$ ESO. Valencia: Castellnou.

Hermi, M. (2017). El análisis del territorio desde una 'totalidad dialéctica'. Más allá de la dicotomía ciudadcampo, de un 'par dialéctico' o de una 'urbanidad rural'. Espaço o Economia, 10. Recuperado de: http://journals. openedition.org/espacoeconomia/2981

Hudault, J. (2011). La protección jurídica del territorio rural. En: E. Muñiz (coord.), Un marco jurídico para un medio rural sostenible (pp. 71-80). Madrid: Ministerio de Medio Ambiente y Medio Rural y Marino.

Jodelet, D. (2009). Recentes desenvolvimentos da noção de representações nas ciências sociais. Em: Â. M. Almeida y Denise Jodelet (orgs.), Interdisciplinaridade e diversidade de paradigmas - Representações sociais (105-122). Brasília: Thesaurus.

Jovchelovitch, S. (2008). Os contextos do saber. Representações, comunidade e cultura. Petrópolis: Vozes.

Kosslyn, S. (1981). El medio y el mensaje en las imágenes mentales: una teoría. Revisión psicológica, 88(1), 46-66.

Liceras, A. (2003). Observar e interpretar el paisaje: Estrategias didácticas. España: Grupo Editorial Universitario.

Limonad, E., y Monte-Mór, R.L. (2012). Por el derecho a la ciudad, entre lo rural y lo urbano. Scripta Nova, Revista Electrónica de Geografía y Ciencias Sociales, vol. XVI, 418 (25). http://www.ub.edu/geocrit/sn/sn-418/sn-418-25. htm 
Lockie, S., Lawrence, G. \& Cheshire, L. (2006). Reconfiguring Rural Resource Governance: The Legacy of Neo-Liberalism in Australia. In: P. Cloke, T. Marsden \& P.H. Mooney (Eds.), The Handbook of Rural Studies (pp. 29-43). London: Sage Publications. https://doi. org/10.4135/9781848608016.n3

Lopes, M.E. (2010). Praxiologia, representação social de menopausa e práticas educativas de enfermeiras na estratégia saúde da família. Tesis doctoral inédita. Río Grande do Norte: Universidade Federal do Río Grande do Norte.

Luis, A. y Urteaga, L. (1982). Estudio del medio y heimatkunde en la geografía escolar. Geocrítica, Cuadernos críticos de geografía humana, 38. Recuperado de: http://www.ub.edu/ geocrit/geo38.htm

Maderuelo, J. (1996). Introducción: el paisaje. En: S. Marchán y J. Maderuelo, Paisaje y pensamiento (pp. 5-10). Huesca: Diputación de Huesca.

Marcén, C. y Molina, P.J. (2006). La percepción del medio ambiente por parte de los escolares. Una visión retrospectiva de 1980 a 2005. En: F. López (coord.), III Jornadas de Educación Ambienta. La educación ambiental en Aragón en los albores del siglo XXI (pp. 1-13). Zaragoza: Departamento de Medio Ambiente. Gobierno de Aragón.

Marco, M. (2012). La voluntad de la mirada: reflexiones en torno al paisaje. Dedica. Revista de Educação e Humanidades, 2, 141-156.

Martínez, J. (1991). Las emociones y su expresión en la primera infancia. Las vías facial y vocal. Revista de Pedagogía de la Universidad de Salamanca, 4, 65-82.

Martínez, M. y Gómez, C.J. (2018). Nivel cognitivo y competencias de pensamiento histórico en los libros de texto de Historia de España e Inglaterra. Un estudio comparativo. Revista de Educación, 379 (enero-marzo), 145-169.

Martínez-Valcárcel, N. (2016). El uso del libro de texto de historia de España en Bachillerato: entre el aula y la casa. História da Educação, 50, 69-93. https://doi. org/10.1590/2236-3459/62453

Mata, M. (1987). Sobre los estudios de geografía agraria en España (1940-1970). Ería, 12, 25-42.

Matthews, H., Taylor, M., Sherwood, K., Tucker, F., \& Limb, M. (2000). Growing-up in the countryside: Children and the rural idyll. Journal of Rural Studies, 16(2), 141-153. https://doi.org/10.1016/S0743-0167(99)00059-5

Méndez, R., Gutiérrez, J. y Guerra, A. (2010). Ciencias Sociales, Geografía. $3^{\circ}$ ESO. Madrid: SM.

Merchán, FJ. (2011). El uso del libro de texto en clase de historia. En: R. López, L. Velasco, V.M. Santidrián y X.A. Armas (coords.), Pensar históricamente en tiempos de globalización (pp. 99-104). Santiago de Compostela: USAC.

Molinero, F. (2019). El espacio rural de España: evolución, delimitación y clasificación. Cuadernos Geográficos 58 (3), 19-56. https://doi.org/10.30827/cuadgeo.v58i3.8643

Molino, S. del (2016). La España vacía. Viaje por un país que nunca fue. Madrid: Turner Noema.

Morales, FJ. (2012). La Geografía de la Percepción: una metodología válida aplicada al caso de una ciudad de tipo medio-pequeño. El ejemplo de Yecla (Murcia). Papeles de Geografía, 55-56, 137-152. https://www.redalyc.org/ pdf/407/40726731012.pdf

Morón, M ${ }^{a}$ C. y Morón, H. (2019). El tratamiento del Medio Rural en el Currículum Oficial de Secundaria en la Última Década. En: X.C. Macía, F.X. Armas y F. Rodríguez (Eds.), La reconfiguración del medio rural en la sociedad de la información. Nuevos desafíos en la educación geográfica (pp. 896-906). Santiago de Compostela: Andavira Editora.

Moscovici, S. (1978). La psychanalyse, son image, son public. Paris: PUF.

Moscovici, S. (2019). Trois présupposés sur les représentations sociales. In : N. Kalampalikis (Ed.), Psychologie des représentations sociales (pp. 9-16). Paris: Editions des Archives contemporaines.

Moscovici, S. y Hewstone, M. (1986). De la ciencia al sentido común. En: S. Moscovici, Psicología Social II. Pensamiento y vida social. Psicología social y problemas sociales (679-710). Barcelona: Paidós.

Muñoz, I. (2012). La ruralidad: análisis de las representaciones del profesorado y propuesta para una enseñanza aprendizaje del compromiso con la ruralidad, aplicada en la región de Maule (Chile). Tesis doctoral inédita. Barcelona: Universitat Autònoma de Barcelona.

Murillo, M. (2003). El uso de los libros de texto en la enseñanza secundaria. Lo que los profesores opinan. Uniciencia, 20 (1), 47-55.

Navia, M. y Estrada, H. (2012). Uso de la técnica de asociación libre para conocer la percepción del consumidor sobre queso costeño en Colombia. Psicogente, 15 (28), 271-286.

Okuda-Benavides, M. y Gómez-Restrepo, C. (2005). Métodos en investigación cualitativa: triangulación. Revista Colombiana de Psiquiatría, XXXIV (1), 118-124.

Onwuegbuzie, A.J., Dickinson, W.B., Leech, N.L. y Zoran, A.G. (2009). A Qualitative Framework for Collecting and Analyzing Data in Focus Group Research. International Journal of Qualitative Methods, 1-21. https:// doi.org/10.1177/160940690900800301

Ortega, J. (2000). Los horizontes de la Geografía. Teoría de la Geografía. Barcelona: Ariel.

Paniagua, A. (2004). La geografía rural, entre el peso de la regulación y las orientaciones constructivistas. Documents d'Anàlisi Geogràfica, 43, 123-134.

Paniagua, Á. y Hoggart, K. (2002). Lo rural, ¿hechos, discursos o representaciones? una perspectiva geográfica de un debate clásico. Globalización y Mundo Rural, 803 61-72.

Parcerisa, A. (1996). Materiales curriculares. Cómo elaborarlos, seleccionarlos y usarlos. Barcelona: Graó.

Pedraza, F. (2011). El drama rural. Metamorfosis de un género. La perspectiva española y el contexto internacional. Vigo: Editorial Academia del Hispanismo.

Peiró, E., Álvaro, N., Vílchez, A. y Farinós, J. (2019). Hacia una nueva cultura territorial desde la educación básica obligatoria. La experiencia de las unidades didácticas "¿qué conozco de mi territorio?" de la Cátedra de Cultura Territorial Valenciana. En: XXVI Congreso de la Asociación Española de Geografía, Crisis y 
espacios de oportunidad. Retos para la Geografía (pp.143158). Valencia: Asociación Española de Geografía y Departamento de Geografía de la Universitat de València.

Pérez, J.A. y Sánchez, M. (2007). Aproximación a las percepciones y orientaciones de los jóvenes ante el futuro del medio rural en Extremadura. Política y Sociedad, 44, 195-217.

Pérez, K., Ezkurdia, G., y Bilbao, B. (2015). El paisaje: un concepto básico en el currículum desarrollado en los libros de texto del País Vasco. Espacio, Tiempo y Educación, 2(2), 225-242. https://doi.org/10.14516/ ete.2015.002.002.011

Pillet, F. (2004). La geografía y las distintas acepciones del espacio geográfico. Investigaciones Geográficas, 34, 141154. https://doi.org/10.14198/INGEO2004.34.07

Prados, M. J. (2009). Naturbanization: New identities and processes for rural -natural areas. In: M. J. Prados (Ed.), Naturbanization: new identities and processes for rural-natural areas (pp. 3 -7). London: Taylor \& Francis Group.

Puente, L. de la (2001). La valoración del conocimiento académico y su incidencia en las aulas de secundaria: los medios rurales y la explicación geográfica del territorio. Nuevos enfoques, nuevas perspectivas de estudio. En: X.M. Souto (Eds.), La Didáctica de la Geografía i la Història en món globalitzat i divers, (pp-206-219). Xàtiva: L'Ullal Edicions i Federaciò de Ensenyament de Comisions Obreres del Pais Valencià.

Rodríguez, A. (2010). Familia rural, valores ambientales y sostenibilidad. Un estudio de educación ambiental en el condado norte de Huelva. En: M. Junyentut y L. Cano (Coords.), Investigar para avanzar en educación ambiental (pp. 181-199). Madrid: Organismo Autónomo Parques Nacionales. Ministerio de Medio Ambiente y Medio Rural y Marino.

Rodríguez, F. (2002). Concebir la geografía escolar desde una nueva perspectiva: una disciplina al servicio de la cultura escolar. Boletín de la Asociación de Geógrafos Españoles, 33, 173-186.

Romero, J. y Farinós, J. (2004). Los territorios rurales en el cambio de siglo. En: J. Romero (coord.), Geografía humana. Procesos, riesgos e incertidumbres en un mundo globalizado (pp.333-394). Barcelona: Ariel.

Romero, J. y Luis, A. (2008). El conocimiento sociogeográfico en la escuela: las tensiones inherentes a la transmisión institucionalizada de cultura y los dilemas de la educación para la democracia en este mundo globalizado. Scripta Nova, Revista Electrónica de Geografía y Ciencias Sociales, XII (270) (123). http://www.ub.edu/geocrit/sn/sn-270/ sn-270-123.htm

Ruiz, A.R., Tula, A.F., y Molinero, F. (2017). La enseñanza de la geografía rural en los estudios universitarios de grado en España: temáticas clave, organización y metodologías de trabajo actuales. Biblio $3 \mathrm{~W}$, Revista Bibliográfica de Geografía y Ciencias Sociales, vol. XXII, 1198. Recuperado de: http://www.ub.edu/geocrit/b3w-1198.pdf

Sá, C. P. (2002). Núcleo central das representações sociais. Petrópolis: Vozes.
Sagoe, D. (2012). Precincts and Prospects in the Use of Focus Groups in Social and Behavioral Science Research. The Qualitative Report, 17(29), 1-16.

Sáiz, J. (2011). Actividades de libros de texto de Historia, competencias básicas y destrezas cognitivas, una difícil relación: análisis de manuales de $1^{\circ}$ y $2^{\circ}$ de ESO. Didáctica de las Ciencias Experimentales y Sociales, 25, 37-64. http:// roderic.uv.es/handle/10550/21329

Sáiz, J. y Colomer, J.C. (2014). ¿Se enseña pensamiento histórico en los libros de texto de Educación Primaria? Clío. History and History teaching, 40, 1-19. http://clio. rediris.es/n40/articulos/saizycolomer2014.pdf

Sammut, G., Andreouli, E., Gaskell, G., \& Valsiner, J. (2015). Social representations: a revolutionary paradigm? In G. Sammut, E. Andreouli, G. Gaskell \& J. Valsiner (Eds.), Cambridge Handbook of Social Representations (pp. 3-11). Cambridge: Cambridge University Press. https://doi. org/10.1017/СBO9781107323650.003

San Pedro, B., y López, I. (2017). El profesorado de ciencias sociales en contextos rurales de Asturias: concepciones y uso de recursos educativos. Aula Abierta, 45, 33-40. https://doi.org/10.17811/rifie.45.2017.33-40

Sánchez, J. (1998). El espacio rural en la enseñanza secundaria: hacia un enfoque más integrador y dinámico. Espacio, Tiempo y Forma. Serie VI, Geografía, t.11,11-29.

Sancho, J. y Reinoso, D. (2012). La delimitación del ámbito rural: una cuestión clave en los programas de desarrollo rural. Estudios Geográficos, Vol. LXXIII, 273, 599-624. https://doi.org/10.3989/estgeogr.201221

Santana, D. (2019). Participación escolar y gestión ambiental. Una dialéctica educativa. Estudio de casos de los parques naturales de la Comunitat Valenciana. Tesis doctoral inédita. València: Universitat de València.

Santiago, J. A. (2011). Educación rural y la enseñanza de la geografía. Sapiens: Revista Universitaria De Investigación, 12(2), 64-76. https://www.redalyc.org/ pdf/410/41030368005.pdf

Santos, M. (1996). A natureza do espaço. São Paulo: Hucitec.

Saraiva, J. (2007). Habitus docente e representaçao social do "ensinar geografia" na Educaçao Básica de TeresinaPiauí. Programa Posgraduaçao em Educaçao. Natal: Universidade Federal Río Grande do Norte.

Segrelles, J.A. (2002). Geografía humana. Fundamentos, métodos, conceptos. Alicante: Editorial Club Universitario.

Silva, E.A. da y Viveiros, K.F.M. (2017). Representações sociais de pobreza construídas pelos cursistas da Especialização Educação, Pobreza e Desigualdade Social realizada no Rio Grande do Norte: primeiros resultados. Educar em Revista, 33 (2), 35-54. https://doi.org/10.1590/01044060.51389

Soja, E. (2008). Postmetrópolis. Estudios críticos sobre las ciudades y las regiones. Madrid: Traficantes de sueños.

Souto, X.M. (1982). Encol do hábitat e do poboamento: o caso de Galicia. Cuadernos de Estudios Gallegos, 98, 7-63.

Souto, X.M. (2006). Geografía de España. 20 curso de Bachillerato. Proyecto Gea-Clío. Valencia: Nau Llibres.

Souto, X.M. (2018). La geografía escolar: deseos institucionales y vivencias de aula. Boletín de la Asociación 
de Geógrafos Españoles, 79, 2757, 1-31. https://doi. org/10.21138/bage. 2757

Souto, X.M. y Ramírez, S. (2002). El trabajo y la didáctica de la geografía e historia. Scripta Nova, Revista Electrónica de Geografía y Ciencias Sociales, vol. VI, 119 (137). Universitat de Barcelona. http://www.ub.es/geocrit/sn/ snl19137.htm

Tesser, C. (2000). Algunas reflexiones sobre los significados del paisaje para la Geografía. Revista de Geografía Norte Grande, 27, 19-26. https://repositorio.uc.cl/ handle/11534/10431

Tonini, I. M., Claudino, S. y Souto, X. M. (2015). Manuais escolares de Geografia de Brasil, Espanha e Portugal: quais as inovações didáticas para o ensino de Geografia? In R. Sebastiá y E.M ${ }^{a}$ Tonda (Coords.), Investigar para innovar en la enseñanza de la Geografía (pp.191-205). Alicante: CEE Limencop.

Valls, R. (2008). La enseñanza de la Historia y textos escolares. Buenos Aires: Libros del Zorzal.

Valls, R. (2012). Historiografía escolar española: siglos XIX-XXI. Madrid: Universidad Nacional de Educación a Distancia.

Vara, J.L. (2010). Un análisis necesario: epistemología de la geografía de la percepción. Papeles de Geografía, 51-52, 337-344.

Vázquez, B. y Aguaded, S. (2001). La percepción de los alumnos de Secundaria de la contaminación: comparación entre un ambiente rural y otro urbano.
En: M. Sánchez y M. Ortega (Eds.), Reflexiones sobre la Didáctica de las Ciencias Experimentales (pp.517-525). Madrid: Universidad Complutense de Madrid.

Vigotsky, L. S. (1993). Pensamiento y lenguaje. Madrid: Aprendizaje Visor.

Vilà, J. y Capel, H. (1970). Campo y ciudad en la geografía española. Madrid: Salvat Editores y Editorial Alianza.

Vilarrasa, A. (2005). El medio local como escala de análisis en la didáctica de las ciencias sociales en educación secundaria. Enseñanza de las ciencias sociales, 4, 11-19.

Vilches, A., Gil, D., Toscano, J.C. y Macías, O. (2014). Desarrollo rural y sostenibilidad. OEI. https://www. researchgate.net/publication/302412565_Desarrollo_ rural_y_Sostenibilidad

Williams, R. (2001). El campo y la ciudad. Buenos Aires: Paidós (Traducción de Alcira Bixio).

\section{NOTA BIOGRÁFICA}

\section{DIEGO GARCÍA MONTEAGUDO}

Profesor Ayudante Doctor. Departamento de Didáctica de las Ciencias Experimentales y Sociales. Universitat de València. Miembro de SOCIAL(S) y del proyecto de renovación pedagógica Gea-Clío. 